Proceedings of the Symposium K: "Complex Oxide Materials for New Technologies" of E-MRS Fall Meeting 2006, Warsaw, September 4-8, 2006

\title{
Dynamics of Thermomagnetic Avalanches in Melt-Textured YBaCuO Superconductors
}

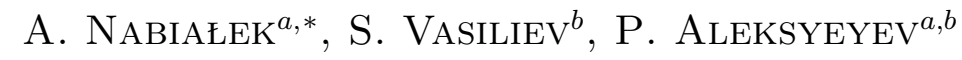 \\ AND V. ChaBANEnKo ${ }^{b}$ \\ ${ }^{a}$ Institute of Physics, Polish Academy of Sciences \\ al. Lotników 32/46, 02-668 Warsaw, Poland \\ ${ }^{b}$ Donetsk Physico-Technical Institute, Ukrainian Academy of Sciences \\ R. Luxemburg str. 72, 83114, Donetsk, Ukraine
}

We investigated dynamics of the thermomagnetic avalanche development in melt-textured samples of the oxide $\mathrm{YBaCuO}$ high temperature superconductor. We found that the flux jumps, caused by the thermomagnetic instabilities, are accompanied by giant convergent oscillations of the surface induction. These results are compared with the dynamics of the flux jumps in other type-II superconductors, including conventional $\mathrm{NbTi}$ and oxide high temperature $\mathrm{Bi}_{2} \mathrm{Sr}_{2} \mathrm{CaCu}_{2} \mathrm{O}_{8+\delta}$ superconductor, and discussed in the framework of the available model of the thermomagnetic avalanche development.

PACS numbers: 74.72.Bk, 74.25.Qt

\section{Introduction}

The magnetic properties of type-II superconductors in external magnetic field are usually described in the model of the critical state [1]. From the viewpoint of thermodynamics, a critical state of the superconductor is metastable. At certain conditions, this critical state may be destroyed by a thermomagnetic avalanche called flux jump [2]. Thermomagnetic avalanches are commonly observed in hard type-II superconductors. From the viewpoint of practical applications of type-II superconductors thermomagnetic avalanches are problematic, because they may drive the superconducting sample into a normal or, at least, into a resistive state. The dynamics of the thermomagnetic avalanche depends on the

*corresponding author; e-mail: nabia@ifpan.edu.pl 
processes of thermal and magnetic diffusion. The equations describing dynamics of the thermomagnetic avalanche are nonlinear, because many of the parameters influencing diffusion processes change nonlinearly with temperature and with the magnetic field. At certain conditions, this may lead to unusual behavior of the thermomagnetic avalanche dynamics, e.g. to the development of dendritic flux structures in superconducting films [3]. In the present work we investigate complex dynamics of thermomagnetic avalanches in samples of oxide high temperature melt-textured $\mathrm{YBaCuO}$ superconductor.

\section{Experiment}

In our studies we investigated two samples of melt-textured $\mathrm{YBaCuO}$ superconductor, both containing approximately $10 \%$ of $\mathrm{BaSnO}_{3}$ phase: a ring $(10 \mathrm{~mm}$ outer diameter, $5 \mathrm{~mm}$ inner diameter, $3 \mathrm{~mm}$ thickness) and a $2.2 \times 5.7 \times 16.6 \mathrm{~mm}^{3}$ slab. The local magnetic induction was measured by miniature Hall probe. The Hall probe measured local induction in the center of the ring, or in the center of the largest surface of the slab. The samples were immersed in liquid helium and the external magnetic field, $\mu_{0} H_{\text {ext }}$, was swept in the range between $-5 \mathrm{~T}$ and $5 \mathrm{~T}$ with the rate of approximately $1 \mathrm{~T} / \mathrm{min}$. The signal from the Hall probe, during the thermomagnetic avalanche development, was registered by a transient recorder (model TCC-1000, Riken Denshi, Co., Ltd.). We performed similar experiments using a $15 \times 2.8 \times 3.1 \mathrm{~mm}^{3}$ slab of conventional NbTi superconductor [4] as well as a $3.3 \times 3.0 \times 0.2 \mathrm{~mm}^{3}$ slab of textured $\mathrm{Bi}_{2} \mathrm{Sr}_{2} \mathrm{CaCu}_{2} \mathrm{O}_{8+\delta}$ [5].

\section{Results}

Figure 1 shows temporal changes of the local induction measured in the center of the $\mathrm{YBaCuO}$ ring, $B_{\text {ring }}$, during one of investigated flux jumps. This flux jump occurred in increasing external magnetic field at $\mu_{0} H_{\text {ext }} \approx 0 \mathrm{~T}$. It is clearly seen that the flux jump is accompanied by giant convergent oscillations of $B_{\text {ring }}(t)$. The amplitude of the oscillations is comparable with the amplitude of the flux jump. This amplitude, however, decreases relatively fast and after the flux jump only several oscillations were registered. Rough estimation of the oscillations frequency gives the value of about $400 \mathrm{~Hz}$. Figure 2 shows dynamics of similar flux jump registered in the case of investigated $\mathrm{YBaCuO}$ slab. In this case one observes about 15 giant oscillations of the surface induction, $B_{\text {surf }}(t)$, whose amplitude is even larger than the jump of the surface induction. A large number of the observed oscillations enabled us to perform spectral analysis, shown in the inset to Fig. 2. One can see that these oscillations occur mainly at the frequency of $540 \mathrm{~Hz}$ and $740 \mathrm{~Hz}$.

Figure 3 shows the result of similar experiment performed in the case of a $15 \times 2.8 \times 3.1 \mathrm{~mm}^{3}$ slab of conventional NbTi superconductor. The flux jump presented in Fig. 3 was registered in increasing external magnetic field, parallel to the $2.8 \mathrm{~mm}$ edge of the slab, at $H_{\text {ext }} \approx 0.4 \mathrm{~T}$ and at the temperature of $3.9 \mathrm{~K}$. In 


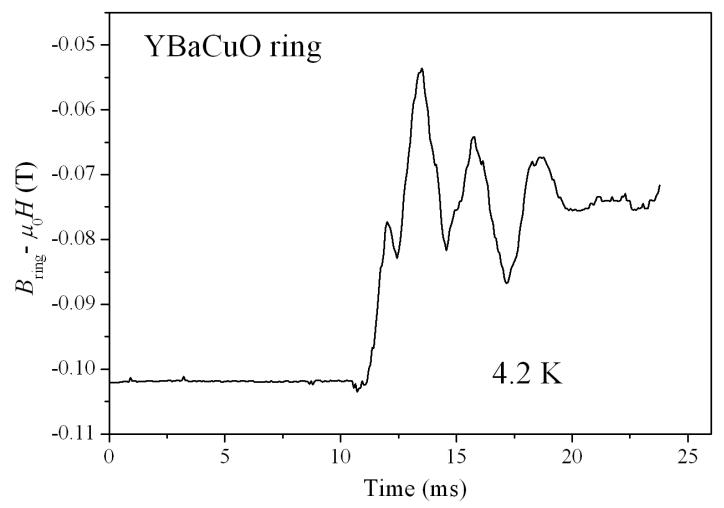

Fig. 1. Temporal changes of the local induction measured in the center of the $\mathrm{YBaCuO}$ ring during one of investigated flux jumps. The flux jump occurred in increasing external magnetic field at $\mu_{0} H_{\mathrm{ext}} \approx 0 \mathrm{~T}$.



Fig. 2. Dynamics of the flux jump in $2.2 \times 5.7 \times 16.6 \mathrm{~mm}^{3} \mathrm{YBaCuO}$ slab. The inset shows spectral analysis of the observed oscillations.

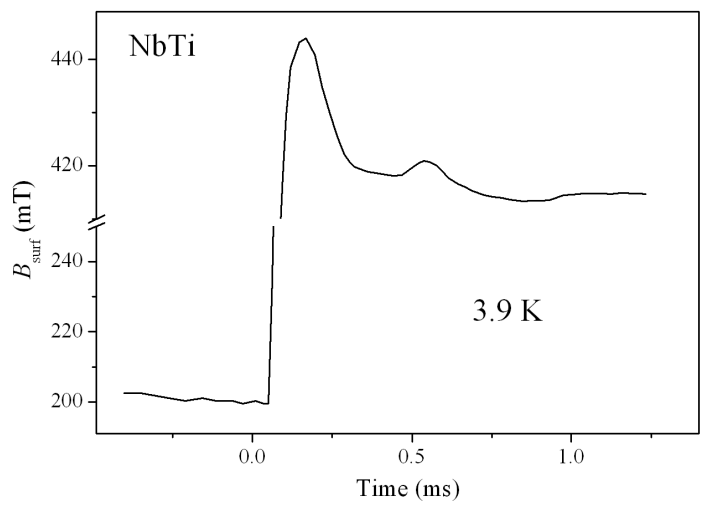

Fig. 3. Dynamics of the surface induction changes of the $15 \times 2.8 \times 3.1 \mathrm{~mm}^{3} \mathrm{NbTi}$ slab during one of the investigated flux jumps. The flux jump occurred in increasing external magnetic field at $\mu_{0} H_{\text {ext }} \approx 0.4 \mathrm{~T}$ and $T \approx 3.9 \mathrm{~K}$. 
the case of NbTi slab we also observe convergent oscillations after the flux jump (frequency about $2.5 \mathrm{kHz}$ ). However, in this case, their amplitude is 1-2 orders of magnitude lower than the amplitude of the flux jump. We also investigated convergent oscillations of the magnetic flux, caused by the flux jumps, in a large $6 \times 15 \times 15 \mathrm{~mm}^{3}$ slab of NbTi superconductor using a pick up coil wound around the investigated sample [6]. The oscillation occurred at the frequency of about $4 \mathrm{kHz}$ and their amplitude was approximately two orders of magnitude lower than the amplitude of the flux jump.

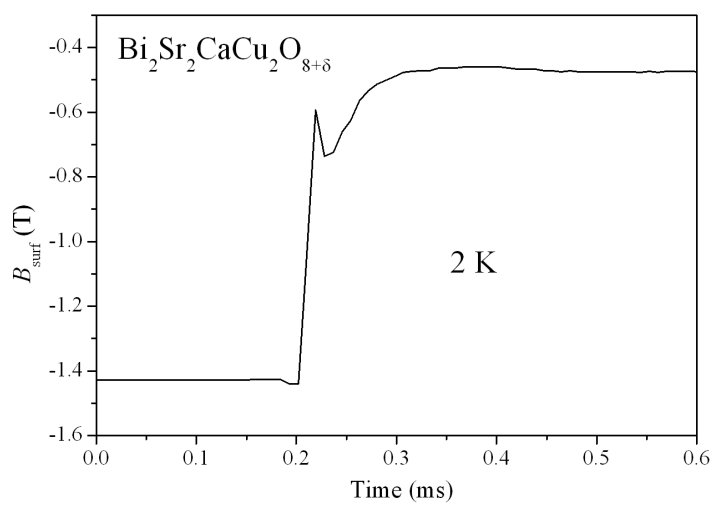

Fig. 4. Dynamics of the surface induction changes of the $3.3 \times 3.0 \times 0.2 \mathrm{~mm}^{3}$ slab of textured $\mathrm{Bi}_{2} \mathrm{Sr}_{2} \mathrm{CaCu}_{2} \mathrm{O}_{8+\delta}$ during one of the investigated flux jumps. The flux jump occurred in increasing external magnetic field (from $-3.5 \mathrm{~T}$ ) at $\mu_{0} H_{\mathrm{ext}} \approx-0.5 \mathrm{~T}$ and $T \approx 2 \mathrm{~K}$

Figure 4 shows the changes of $B_{\text {surf }}(t)$ during one of the investigated flux jumps, taken at the surface of $3.3 \times 3.0 \times 0.2 \mathrm{~mm}^{3}$ slab of textured $\mathrm{Bi}_{2} \mathrm{Sr}_{2} \mathrm{CaCu}_{2} \mathrm{O}_{8+\delta}$. This jump was registered in increasing external magnetic field (from $-3.5 \mathrm{~T}$ ) at $\mu_{0} H_{\text {ext }} \approx-0.5 \mathrm{~T}$ and $T=2 \mathrm{~K}$. In the case of $\mathrm{Bi}_{2} \mathrm{Sr}_{2} \mathrm{CaCu}_{2} \mathrm{O}_{8+\delta}$ sample we did not observe oscillations like in the case of previous samples, but also in this case the surface induction changes non-monotonically with time. One of possible interpretations of the observed in Fig. 4 peak is an oscillation of a very short decay time, shorter than the period of the oscillation.

\section{Discussion}

Our experimental results show that in the case of many different type-II superconductors during the thermomagnetic instability development surface induction changes non-monotonically with time. In the case of $\mathrm{YBaCuO}$ samples giant convergent oscillations were observed.

In order to analyze the dynamics of the thermomagnetic avalanches theoretically, it is necessary to solve a set of the Maxwell equations and the equation of heat diffusion. Analytically, it can be done only in linear approximation, it 
means, at the initial stage of the thermomagnetic avalanche development. At this stage the theory predicts the possibility of occurrence of the temperature and electromagnetic field oscillations [2]. Such oscillations have been observed in many experimental works (see Ref. [2] for a review). The amplitude and frequency of these oscillations depends strongly on the properties of investigated samples.

Our experimental studies show that oscillations of electromagnetic field can also occur at the final stage of the thermomagnetic avalanche and that the amplitude of these oscillations may be, at certain conditions, comparable or even larger than the amplitude of the observed flux jumps. The amplitude and frequency of the oscillations, at the final stage of the avalanche, also depend strongly on the properties of the investigated samples.

One of the important parameters influencing the dynamics of the thermomagnetic avalanche development is the relation between the thermal and magnetic diffusivity. In our experimental conditions one can assume the conventional superconductor, $\mathrm{NbTi}$, to be in the flux flow regime. In the flux flow regime, because of relatively large flux flow resistivity, it is reasonable to assume the magnetic diffusivity to be larger than the thermal one. On the other hand, these assumptions are not true in the case of high temperature superconductors, which are characterized by a strong flux creep even at the temperature of liquid helium. The conditions of the thermomagnetic avalanche development are also dramatically influenced by the sample shape [7]. The shapes of our samples were different.

The above discussed parameters are, in our opinion, responsible for different magnetic flux dynamics in different samples studied in our experiments. Unfortunately, the oscillations observed in our experiment, at the final stage of the thermomagnetic avalanche, cannot be analyzed in the framework of the linear model, presented in Ref. [2], because many of the parameters, influencing the observed phenomena, change nonlinearly during the flux jumps. In order to analyze these phenomena in more details numerical simulations are necessary.

\section{Conclusions}

The oxide high temperature superconductors reveal very complex dynamics of the thermomagnetic avalanche development. In the case of melt-textured $\mathrm{YBaCuO}$ sample one observes giant convergent oscillations of the surface induction at the final stage of the thermomagnetic avalanche. The amplitude of these oscillations is comparable, or even higher, than the amplitude of the flux jumps.

\section{References}

[1] C.P. Bean, Phys. Rev. Lett. 8, 250 (1962).

[2] R.G. Mints, A.L. Rakhmanov, Rev. Mod. Phys. 53, 551 (1981).

[3] T.J. Johansen, M. Baziljevich, D.V. Shantsev, P.E. Goa, Y.M. Galperin, W.N. Kang, H.J. Kim, E.M. Choi, M.S. Kim, S.I. Lee, Europhys. Lett. 59, 559 (2002). 
[4] V.V. Chabanenko, V.F. Rusakov, A. Nabialek, S. Piechota, S. Vasiliev, H. Szymczak, Physica C 369, 77 (2002).

[5] A. Nabialek, V.V. Chabanenko, V. Rusakov, S. Vasiliev, H. Szymczak, S. Piechota, H. Dabkowska, A. Dabkowski, B. Gaulin, M. Niewczas, O. Mironov, J. Low Temp. Phys. 130, 425 (2003).

[6] V.V. Chabanenko, V.K. Rusakov, V.A. Yampol'skii, H. Szymczak, S. Piechota, S. Vasiliev, A. Nabialek, Low Temp. Phys. 28, 387 (2002).

[7] D.V. Denisov, A.L. Rakhmanov, D.V. Shantsev, Y.M. Galperin, T.H. Johansen, Phys. Rev. B 73, 014512 (2006). 